\title{
Premature Hair Greying - Magnitude and Associated Factors: A cross-sectional study in a university in Mysuru \\ Shrey Kansal ${ }^{1}$, Anil S Bilimale ${ }^{2}$, Arun Gopi ${ }^{3}$, Sushma BV ${ }^{4}$
}

${ }^{1}$ MBBS, JSS Medical College, JSS AHER, Mysuru, Karnataka; ${ }^{2}$ Assistant Professor, Department of Community Medicine, JSS Medical College, JSS AHER, Mysuru, Karnataka; ${ }^{3}$ Lecturer, Department of Community Medicine, JSS Medical College, JSS AHER, Mysuru, Karnataka; ${ }^{4}$ Assistant Professor, Department of Nutrition \& Dietetics - Faculty of Life Sciences, JSS Academy of Higher Education and Research, Mysuru, Karnataka

\begin{tabular}{|c|c|c|c|c|c|c|c|c|}
\hline Abstract & Introduction & Methodology & Results & Conclusion & References & Citation & \multicolumn{2}{|c|}{ Tables / Figures } \\
\hline \multicolumn{9}{|c|}{ Corresponding Author } \\
\hline \multicolumn{8}{|c|}{$\begin{array}{l}\text { Dr Anil S Bilimale, Assistant Professor, Department of Community Medicine and School of Public Health, JSS } \\
\text { Medical College, JSS AHER, Mysuru, Karnataka } 570015 \\
\text { E Mail ID: anilbilimale@gmail.com }\end{array}$} & 回的品 \\
\hline
\end{tabular}

\section{Citation}

Kansal S, Bilimale AS, Gopi A, Sushma BV. Premature Hair Greying - Magnitude and Associated Factors: A cross-sectional study in a university in Mysuru. Indian J Comm Health. 2021;33(3):462-465. https://doi.org/10.47203/IJCH.2021.v33i03.010 Source of Funding: ICMR STS project 2019-05240 Conflict of Interest: None declared

\section{Article Cycle}

Received: 06/06/2120; Revision: 05/08/2021; Accepted:11/09/2021; Published:30/09/2021

This work is licensed under a Creative Commons Attribution 4.0 International License.

\section{Abstract}

Background: Premature hair greying (PHG) refers to the diffuse loss of hair color at an age earlier than that is generally accepted as physiological. Studies have found that it affects self-esteem and social life. Our efforts were to estimate the prevalence among students <25years and understand the associated factors. Design and Methods: A cross-sectional study using a simple random sampling technique was used to survey 358 students at a university in Mysuru. The data were analyzed using SPSS v22. The association and relationship of PHG with attributed risk factors and various socio-clinical factors have been analyzed using Chi-Square Test, Independent sample t-test, and Mann Whitney U-test. A p-value of less than 0.05 has been considered statistically significant. Results: In this study, the prevalence of PHG was $28.2 \%$. We observed that PHG was associated with male gender, obesity, and positive paternal, maternal, and family history of PHG. The relation of PHG with lower dietary iron intake, tobacco smoking amount, and frequency was also found in this study. However, no association between PHG and psychological stress, alcohol consumption, dietary Vitamin B12, and D intake, shampoo, and oil usage frequency was found. Conclusion: We recommend that further studies should be done to check if weight reduction, smoking cessation, and maintenance of dietary iron adequacy can help in preventing PHG.

\section{Keywords}

Obesity; Alcohol Drinking; Risk Factors; Surveys and Questionnaires; Tobacco Smoking; Stress; Psychological

\section{Introduction}

Premature Hair Greying (PHG) refers to the diffuse loss of hair colour at an earlier age than that is generally accepted as physiological, i.e., before the age of 25 years in Asians. $(1,2,3,4)$. Studies have shown that PHG might be a risk marker for various systemic diseases like Coronary Artery Disease. (3) Moreover, since hair has a vital role in people's social and sexual communication, PHG can be distressing, especially for adolescents affecting their socio-cultural spheres of life and, in turn, their mental and social health. $(5,6)$

PHG is a rising concern for children and their parents, making it essential to evaluate its prevalence and predominating risk factors. $(1,2)$
The exact aetiology of PHG remains unknown, but it is considered that it occurs in genetically predisposed people exposed to various socio-clinical factors that increase oxidative stress and damage to melanocytes. PHG has also been linked to deficiencies of various nutrients (e.g., iron, vitamin B12, and D) because of their involvement in melanogenesis. $(1,7)$. However, studies investigating various factors that influence PHG in a large population remain scarce, especially in India. $(2,3,4)$

Since, inadequate usage of shampoo and oil on hair and scalp has often been perceived as a risk factor of PHG, this study also explores the relation between PHG and shampoo and oil usage frequency. (5) 


\section{Aims \& Objectives}

To evaluate the prevalence of PHG and identify the association and relation of $\mathrm{PHG}$ with plausible predominating socio-clinical risk factors and nutritional deficiencies

\section{Material \& Methods}

A cross-sectional study was conducted over two months from August to September 2019 at a University in Mysuru. The respondents were students of the University, aged below 25 years, chosen through a simple random sampling technique. Informed consent was taken from each respondent. The exclusion criteria were the refusal to participate in the study. Assuming the prevalence of PHG in the university as $26 \%$, taking precision of $5 \%$, and an alpha level of $5 \%$, the minimum sample size of 296 was required for the study and eventually, a sample size of 358 was used in this study. (2)

Data was collected through a self-administered questionnaire. The questionnaire could be answered online or offline as per the convenience of respondents. Statistical analysis was done using SPSS v22. A significance level of $95 \%$ was chosen.

The respondents were asked if they have grey hair on their bodies (scalp or any other region). Based on their responses, the prevalence of self-reported PHG was calculated. The information on anthropometric measures; height and weight of the respondent was used to estimate the Body Mass Index (BMI), as a surrogate measure of body fat of the respondent. Respondents with a BMI greater than or equal to 25 were classified as obese and respondents with a $\mathrm{BMI}$ of less than 25 were classified as non-obese, as per the Asia-Pacific classification of BMI.(8) Information on family history includes the history of PHG in father, mother, and siblings, paternal, and maternal grandparents. Responses of those respondents who were not sure of the presence of PHG in their family members were excluded from the evaluation of this association. Respondents were assessed for their average daily dietary Vitamin B12 and D (in $\mu \mathrm{g} /$ day), and iron intake (in $\mathrm{mg} /$ day), using 24-hr dietary recall for 3 consecutive days; Saturday, Sunday, and Monday. This data was obtained by periodically interviewing 154 respondents chosen at random from the surveyed population. Since different diet patterns/ habits (vegetarian diet, non-vegetarian diet, eggetarian diet, and vegan diet) differ in the composition of these nutrients, respondents were also asked about their dietary habits.

The frequency and amount of tobacco smoking were measured as packs per year (PPY) (a pack consists of 20 cigarettes). The alcohol intake of respondents was measured in 'unit alcohol/ month'. Stress levels of respondents were assessed using the ten-item Cohen's Perceived Stress Scale (PSS) which comprises 10 questions. (9) The shampoo and oil usage frequency of respondents were calculated in 'usage frequency/ week'.
Associations between PHG and parameters like; obesity, gender, and family history of PHG were evaluated using the Chi-square test. Relationships between PHG and parameters like BMI, PSS scores, dietary intake of Iron, Vitamin B12, and Vitamin D were evaluated by comparing means using an independent sample t-test since these parameters were found to be normally distributed in the population. The relationship of PHG with alcohol intake, smoking amount and frequency, shampoo, and oil usage frequency was evaluated by comparing medians using the Mann-Whitney $U$ test since these parameters were not found to be normally distributed in the study population. Ethical approval: From institutional ethical committee JSSMC/ IEC/ 1405/ 10NCT/ 201920

\section{Results}

The mean age of the respondents was $19.4 \pm 1.6$ years.

Prevalence and Gender: The prevalence of self-reported PHG in the studied population was found to be $28.2 \%$ $(n=101)$. On performing the Chi-Square test, it was found that the prevalence of PHG was significantly higher $(p=0.008)$ in males $(36.2 \%, n=50)$ than in females $(23.1 \%$, $n=51)$.

BMI and Obesity: Through independent sample t-test, the average BMI of respondents with PHG (mean=23.7, $S D=3.8)$ was significantly higher $(p=0.008)$ than the average $\mathrm{BMI}$ of respondents without $\mathrm{PHG}$ (mean=22.2, $S D=4.08)$. Furthermore, on using the Chi-square test, the prevalence of PHG was found to be significantly more $(p=0.002)$ in obese respondents $(41.1 \%, n=37)$ than in non-obese respondents $(23.9 \%, n=64)$.

Family History: [Table 1] shows the positive history of PHG in father, mother, or any of the family member/s (includes parents, siblings, paternal and maternal grandparents). Using the chi-square test it was found that positive paternal, maternal, and family history of PHG was significantly more common $(p<0.001, p=0.004, p<0.001$, respectively) in respondents with PHG than in respondents without PHG.

Tobacco Smoking: On comparing median 'packs per year (PPY) of respondents having PHG (median=0, IQR=0, mean=0.06, $S D=0.48$ ) and respondents without $P H G$ (median=0, IQR=0, mean=0.02, SD=0.13) using Mann Whitney $U$ test, a statistically significant difference was found between the 2 medians ( $p=0.025)$.

Alcohol Consumption: On performing Mann Whitney $U$ test, the difference in medians of alcohol intake (in units/month) of respondents with PHG (median =0, $I Q R=0$, mean=0.69, $S D=1.60$ ) and respondents without PHG (median=0, IQR=0, mean=0.77, SD=2.03) was found to be insignificant $(p=0.68)$.

Shampoo and Oil Usage: No significant difference $(p=0.82)$ between median shampoo usage frequency (in usage frequency/week) of respondents with PHG (median=2, IQR= 2, mean=2.42, SD=1.59) and respondents without PHG (median=2, $I Q R=1$, mean=2.51, 
$S D=2.51$ ) was observed on performing Mann Whitney $U$

Test. Hence, no relationship was observed between shampoo usage frequency and PHG. Similarly, Mann Whitney $U$ test showed that the difference between median hair oil usage frequency (in usage frequency/month) in respondents with $\mathrm{PHG}$ (median=4, $\mathrm{IQR}=6$, mean=6.19, $\mathrm{SD}=6.71$ ) and without $\mathrm{PHG}$ (median=4, $\mathrm{IQR}=6$, mean=5.91, $S D=7.96)$ is statistically insignificant $(p=0.94)$.

Nutrition and Diet: Mean dietary intake of Iron (in $\mathrm{mg} /$ day) was significantly lower $(\mathrm{p}=0.0004)$ in respondents with PHG (mean=17.16, SD=2.52) than in respondents without PHG (mean=18.96, SD=3.00) as per independent sample t-test. However, no significant difference $(p>0.99)$ in mean dietary vitamin B12 intake (in $\mu \mathrm{g} /$ day) in respondents with PHG (mean=0.73, SD=0.20) and without PHG (mean=0.69, SD=0.16) was found through independent sample t-test. Similarly, the difference between mean vitamin $D$ intake (in $\mu \mathrm{g} /$ day) in respondents with PHG (mean=5.92, SD=1.91) and respondents without PHG (mean=6.17, SD=1.91) was also found to be insignificant $(p=0.36)$ as per independent sample t-test. Table 2 shows that there is no association between dietary habits and PHG as per the chi square test. Psychological Stress: On performing the Mann Whitney U test, the difference between median PSS scores of respondents with PHG (median=20, IQR=9) and respondents without PHG (median 20, IQR=6) was found to be insignificant.

\section{Discussion}

The prevalence of self-reported PHG in students at a University in Mysuru was found to be $28.2 \%$. The prevalence obtained is near to the value reported by TS et al., Shin et al, and Belli et al. but are higher in comparison to the values reported by Bhat et al., and Francis et al.. $(1,2,4,3,10)$ The difference in the prevalence of PHG obtained in different studies can be attributed to differences in demographics of the target population, place of study, inclusion and exclusion criteria, and differences in various environmental and social factors in different regions.

This study showed a significant association between gender and PHG with it being significantly more common in males. The results of this study differ from other studies done by Bhat et al. and Belli et al. which found no genderspecific predilection in the prevalence of PHG indicating that more studies are required to be done in different regions of the world to establish if the gender of a person has any role in PHG. $(1,3)$

Literature gives different results for the association between obesity and PHG. For example, a study conducted by Shin et al. has found association whereas, Belli et al. concluded on the contrary. $(4,3)$ In this study, PHG is found to be related to BMI and is associated with obesity. The result aligns with the understanding that obesity can lead to decreased melanogenesis because leptin resistance often observed in obese people increases the secretion of melanocyte-stimulating hormone (MSH) antagonists. $(4,11)$

Psychological stress is a well-known contributor to Systemic Oxidative Stress and hence a possible contributor to the pathogenesis of PHG. In this study, the relationship between psychological stress and PHG was not found to be statistically significant in the study population. However, the relationship between perceived stress and PHG was found in studies conducted by TS et al. and Belli et al. probably because of their larger sample size. $(2,3)$

This study showed a strong association of PHG with paternal, maternal, and family history of PHG. The results of this study are in agreement with those obtained in studies conducted by TS et al., Belli et al., Shin et al., and Daulatabad et al. $(2,3,4,6)$ which also reported a strong association between PHG and family history of PHG, thereby, indicating a strong genetic component involved in the aetiopathogenesis of PHG. However larger and more elaborate studies must be done to see if there is any definite pattern of inheritance of PHG.

Smoking is a larger contributor to Oxidative Stress, hence; it might be playing an important role in the aetiopathogenesis of PHG in smokers making it a possible risk factor. This study showed that PHG is related to higher smoking amount and frequency. This is in conformation with the results obtained in the studies conducted by Shin et al., Zayed et al., and TS et al. $(4,11,2)$

This study showed no relation between alcohol consumption and PHG. The result of this study is contradictory to that obtained in the study conducted by Belli et al., which showed that alcohol consumption frequency was significantly higher in respondents having PHG because alcohol is a known contributor to oxidative stress. (3) The relation between PHG and alcohol consumption was not found in the study probably because most of the respondents were below the age of 21 years which is the legal age of drinking in the region as per the government rules.

Many people perceive a lack of self-care of scalp and hair in the form of inadequate washing of hair/ scalp with shampoo or inadequate oiling of the scalp as risk factors of PHG. Even though no scientific basis for the same could be found, a study conducted by Saad et al. found that $44.3 \%$ of subjects perceived that lack of self-care in the form of inadequate washing, inadequate oiling of the scalp, or excessive use of cosmetics is a risk factor of PHG. (5) This study showed no relation to PHG with shampoo usage and oil usage frequency debunking the myth.

PHG has often been linked to various nutritional deficiencies because of the involvement of certain nutrients in the melanogenesis of hair follicles (e.g. iron) and cell proliferation in hair follicles (e.g. vitamin B12 and folic acid). $(1,7)$ In this study, the relation between PHG 
and dietary Iron intake was found. The mean dietary intake of respondents with PHG being significantly lesser than the mean dietary intake of respondents without PHG. However, no relation between PHG and dietary Vitamin D and B12 intake was found. To our knowledge, this is the first study to evaluate the relation between PHG and dietary intake of Iron, Vitamin B12 and Vitamin D. However study conducted by Chakrabarty et al. found a relation between PHG and serum ferritin and Vitamin B12 levels and no relation between PHG and serum Vitamin D3 levels. (7) Another study conducted by Bhat et al. found out the relation between PHG and serum ferritin and Vitamin D3 levels. (1)

Since different dietary habits (like vegetarianism, nonvegetarianism) usually differ in quantity and proportions of these nutrients, this study attempted to find out if there was any association between PHG and different dietary habits/ patterns. However, no such association was found between PHG and different dietary habits; vegetarian, non-vegetarian, eggetarian, and vegan diet. This relates to the study conducted by Belli et al. which found no association between PHG and different dietary habits. (3)

\section{Conclusion \& Recommendation}

The results of this study shall help to plan preventive measures for PHG and serve as a reference for further larger studies that can be conducted at the regional level and national level which are truly representative of the general population.

As this study showed a relationship/ association of PHG with low dietary iron intake, obesity, and smoking, further studies with advanced study designs and sampling techniques should be conducted to determine the causality of associated risk factors.

\section{Limitation of the study}

As self-administered questionnaire, there was a possibility of recall bias, under-reporting due to the non-observance of PHG. Furthermore, the study shows relationships and associations of attributed risk factors with PHG but does not prove any causal relationship. The generalizability of the findings is limited.

\section{Relevance of the study}

PHG is found to be associated with male gender, higher BMI, obesity, positive paternal, maternal, and family history of PHG, lower dietary iron intake, tobacco smoking

\section{Tables}

TABLE 1 PREVALENCE OF A POSITIVE HISTORY OF PHG IN A FAMILY OF RESPONDENTS

\begin{tabular}{|l|l|l|}
\hline $\begin{array}{l}\text { Positive } \\
\text { History }\end{array}$ & $\begin{array}{l}\text { Respondents } \\
\text { PHG }\end{array}$ & $\begin{array}{l}\text { With } \\
\text { Respondents Without }\end{array}$ \\
\hline Paternal & $27.1 \%(n=19)$ & $6.75 \%(n=15)$ \\
\hline Maternal & $19.2 \%(n=15)$ & $7.69 \%(n=18)$ \\
\hline Family & $55.0 \%(n=49)$ & $21.7 \%(n=53)$ \\
\hline
\end{tabular}

amount and frequency. However, no association between PHG and psychological stress, alcohol consumption, dietary Vitamin B12, and D intake, shampoo, and oil usage frequency was found.

\section{Authors Contribution}

All authors have contributed equally.

\section{Acknowledgement}

ICMR STS project for its support, Ms. Mandvi Vashishta and Mr. Sabi Saji Simon for assisting in data collection, community Medicine department faculties and students for cooperating in the study.

\section{References}

1. Bhat RM, Sharma R, Pinto AC, Dandekeri S, Martis J. Epidemiological and Investigative Study of Premature Graying of Hair in Higher Secondary and Pre-University School Children. Int J Trichology. 2013; 5(1):17-21.

2. Ts B, Sathyanarayana BD, Swaroop MR, Devaraj Y, Jc R, Dukkipati M, et al. A clinicoepidemiological study of premature canities of degree college students in the rural. International Journal Of Advances In Case Reports.2016; 3(14):489-493

3. Belli AA, Etgu f, Gok So, Kara B, Dogan G. Risk Factors for Premature Hair Graying in Young Turkish Adults. Pediatric Dermatology - Wiley Online Library. 2016; 33(4):232-236.

4. Shin H, Ryu HH, Yoon J, Jo S, Jang S, Choi M et al. Association of premature hair graying with family history, smoking, and obesity: A cross-sectional study. Journal of American Academy of Dermatology. 2015; 72(2):321-327.

5. Saad M, Babar NF, Majeed R, Rehman AU, Khan OA, Chatha DE, et al. Impact of Premature Greying of Hair on Socio-cultural Adjustment and Self-esteem among Medical Undergraduates in Foundation University, Islamabad. Cureus. 2019; 11(7):e5803.

6. Daulatabad D, Grover C, Singal A. Quality of life and psychological impact of premature canities: A study from North India. Pigment Int. 2016; 3(1):24.

7. Chakrabarty S, Krishnappa P, Gowda DG, Hiremath J. Factors Associated with Premature Hair Graying in a Young Indian Population. Int J Trichology. 2016; 8(1):11-14.8.

8. Pan WH, Yeh WT. How to define obesity? Evidence-based multiple action points for public awareness, screening, and treatment: an extension of Asian-Pacific recommendations. Asia Pac J Clin Nutr. 2008; 17(3):370-374

9. Cohen S, Kamarck T, Mermelstein R. Perceived Stress Scale [Internet]. American Psychological Association; 2014 [cited 2020 May 16]. Available from: http://doi.apa.org/getdoi.cfm?doi=10.1037/t02889000

10. Francis DA, George DRM, V DMNK. Study of prevalence of premature graying of hair among school-going children aged between 5-15 years. Indian J Appl Res. 2019; 9(8):50-52.

11. Morpurgo G, Fioretti B, Catacuzzeno L. The increased incidence of malignant melanoma in obese individuals is due to impaired melanogenesis and melanocyte DNA repair. Medical Hypotheses. 2012; 78(4):533-535.

12. Zayed AA, Shahait AD, Ayoub MN, Yousef AM. Smokers' hair: Does smoking cause premature hair graying?. Indian Dermatol Online J. 2013;4(2):90-92. doi:10.4103/2229-5178.110586.

TABLE 2 ASSOCIATION OF DIETARY HABITS AND PHG IN RESPONDENTS

\begin{tabular}{|l|l|l|l|}
\hline Dietary habit & $\begin{array}{l}\text { Respondents } \\
\text { With PHG }\end{array}$ & $\begin{array}{l}\text { Respondents } \\
\text { Without PHG }\end{array}$ & $\begin{array}{l}\text { p- } \\
\text { value }\end{array}$ \\
\cline { 1 - 3 } Vegetarian & 15 & 44 & \multirow{2}{*}{0.389} \\
\hline Non-vegetarian & 76 & 185 & \\
\hline Eggetarian & 8 & 27 & \\
\hline Vegan & 2 & 1 & \\
\hline
\end{tabular}

